\title{
Mesures de la quantité relative de feuillage par strates dans des taillis de chêne pubescent
}

\author{
Claudie HOUSSARD et J. ESCARRE \\ Centre d'Etudes phytosociologiques et écologiques Louis Emberger, C.N.R.S. \\ Département d'Ecologie générale, B.P. 5051, F 34033 Montpellier Cedex
}

\begin{abstract}
Résumé
Trois méthodes permettant de construire des profils verticaux de feuillage en forêt sont décrites et les résultats obtenus avec chacune d'elles sont comparés. Ces méthodes sont :

1) la méthode des photographies hémisphériques pour estimer l'indice foliaire ;

2) la méthode des points-quadrats optiques pour évaluer la quantité de feuillage qui exprime un nombre moyen de contacts de feuilles avec des lignes de visée verticales virtuelles;

3) une méthode radiométrique qui utilise les propriétés sélectives d'absorption spectrale du rayonnement solaire par la végétation. Un rapport, $K$, de deux tensions proportionnelles aux intensités spectrales des radiations transmises rouges $(679 \mathrm{~nm})$ et infra-rouges $(798 \mathrm{~nm})$ est mesuré.

Ces méthodes ont été testées dans quatre taillis de Chêne pubescent (Quercus pubescens Willd.) des Hautes Garrigues du Montpelliérais.

Il en résulte que les valeurs absolues de l'indice foliaire, de la quantité de feuillage ou du rapport $\mathrm{K}$ sont affectées par la structure de la végétation dans les stations ou par les protocoles de recueil des données; mais dans une station, les trois méthodes sont corrélées et les profils d'indice foliaire ou de quantité de feuillage, en valeurs relatives sont parfaitement comparables. Le choix d'une méthode fait intervenir le coût de recueil des données. C'est la méthode des points-quadrats optiques qui est la plus rapide et qui fournit le plus d'informations pour obtenir des profils verticaux de feuillage en forêt.
\end{abstract}

\section{1. - Introduction}

L'architecture et la stratification des voûtes forestières ont une grande influence sur les interactions entre les plantes et l'environnement au travers des modifications des flux de rayonnement, de gaz, d'eau et d'éléments nutritifs (MoNSI \& SAEKI, 1953; CACHAN, 1963 ; KIRA et al., 1969). Leurs caractéristiques sont déterminantes sur les facteurs de sélection de l'habitat par les animaux. Il en est ainsi pour les oiseaux, par exemple (Mac Arthur \& Mac Arthur, 1961; Mac Arthur et al., 1966; Cody, 1968 ; Blondel et al., 1973), et les cervidés (WebB, 1948 ; Moen, 1973). 
Ainsi, que ce soit pour des études de la production primaire ou pour l'étude de l'organisation des communautés animales ou végétales, il est important de reconstituer les profils verticaux de la nappe foliaire et de localiser les niveaux de plus forte densité de feuilles.

L'établissement de ces profils peut se faire par recueils de feuilles par strates (MONSI \& SAEKI, 1953; HaRcombe \& MARKS, 1977), ou par la méthode des pointsquadrats (WARREN-WiLson, 1963 ; Miller, 1967, 1969 ; Ford \& Newbould, 1971).

Ces méthodes ont le désavantage d'être soit destructives, soit d'être très difficiles à mettre en place (utilisation de tours métalliques, etc.) et pratiquement impossible à utiliser pour étudier des voûtes foliaires très hautes.

Par contre trois méthodes nous sont apparues d'un emploi plus aisé. Ce sont :

1) la méthode d'estimation de l'indice foliaire à l'aide des photographies hémisphériques ;

2) la méthode de mesure de la quantité de feuillage à l'aide de points-quadrats optiques ;

3) la méthode radiométrique qui utilise les propriétés d'absorption spectrale sélective du rayonnement solaire par les feuilles, et qui permet d'estimer indirectement la quantité de feuillage.

Le propos de cet article est de décrire les trois méthodes et de comparer les résultats obtenus avec chacune d'elles. Elles ont été testées dans quatre taillis de Chêne pubescent (Quercus pubescens Willd.) des Hautes Garrigues du Montpelliérais (HOUSSARD, 1979).

\section{2. - Site}

Les communautés étudiées se situent dans la région des Hautes Garrigues du Montpelliérais au nord de Montpellier.

Les formations boisées de cette zone sont constituées par le Chêne vert (Quercus ilex L.) ou le Chêne pubescent (Quercus pubescens Willd.) ou par des mélanges de ces deux espèces. La région a été décrite par les phytosociologues comme une région de transition entre le Quercetum ilicis galloprovinciale Br.-Bl. (1915) 1936, et le Querceto-Buxetum Br.-Bl. (1915) 1931 (BRAUN-BlanQueT, 1936 ; Braun-BlanqueT et al., 1952).

Quatre stations, qui se situent dans les taillis de Chêne pubescent, ont été échantillonnées. Ce sont Rouet, La Rouvière 1, La Rouvière 2 et Murviel. Elles ont été choisies selon un gradient de hauteur de voûte (Rouet, voûte basse ; Murviel, voûte plus haute ; La Rouvière 1 et 2 , voûte à hauteur intermédiaire). Le tableau 1 présente leurs caractéristiques.

Dans ces quatre stations, les données ont été recueillies systématiquement le long d'une ligne horizontale de 32 mètres de longueur. Les points de récolte des mesures sont espacés régulièrement le long de la ligne. 


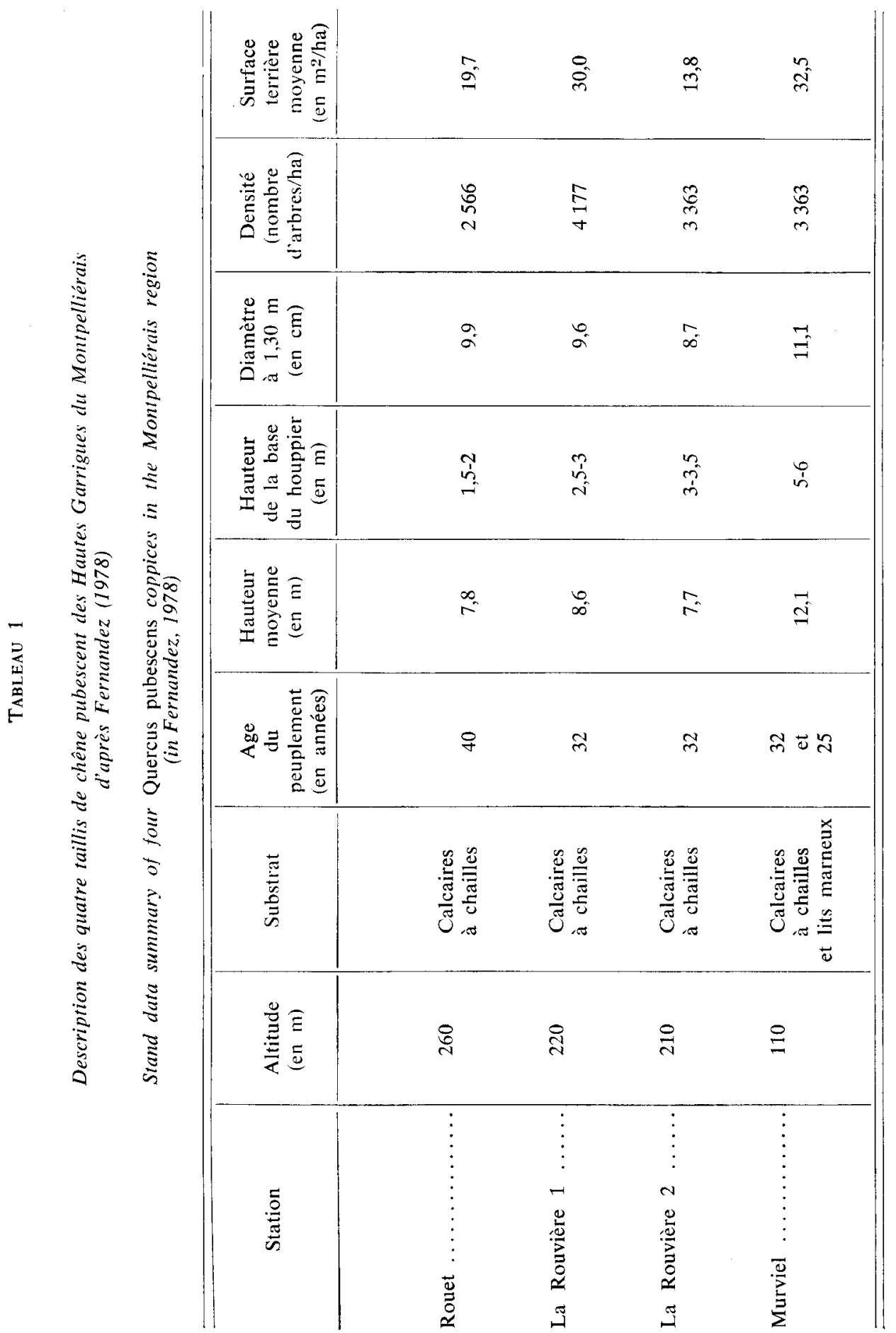




\section{3. - Description des méthodes}

\section{1. - Méthodes d'estimation de l'indice foliaire \\ à l'aide de photographies hémisphériques}

\subsection{Modèle}

WARREN-WILSON (1959, 1960, 1963) estime l'indice foliaire à l'aide d'une méthode de points-quadrats inclinés; la liaison entre les résultats obtenus à l'aide de cette méthode et la probabilité de passage d'un rayon lumineux incliné sur l'horizontale à travers une couche végétale d'indice foliaire f, est évidente (ANDERSON, 1966 ; WARREN-WILSON, 1967 ; NILSON, 1971 ; LEDENT, 1974, 1976).

En particulier, sur une photographie hémisphérique prise au sein d'une formation végétale, on peut mesurer pour des hauteurs de visée, 1, au-dessus de l'horizon, la part de rayons lumineux (trous) qui traverse la voûte végétale sans interception (Ducrey, $1975 \mathrm{a}$ et $\mathrm{b}$; BonHomme, 1974, 1976).

Chartier (1966) montre que l'on a une relation entre la part de ciel vue à travers le couvert végétal, $s_{1}$, pour une hauteur de visée, et l'indice foliaire, $f$, de la culture surplombant le niveau de mesure du type :

$$
\mathrm{s}_{1}=\mathrm{e}^{-\mu(1,1)} \cdot \mathbf{1}
$$

avec $\mu(\mathrm{i}, 1)$ : fonction d'extinction qui dépend de la hauteur de visée (1), des fréquences d'inclinaison (i) des feuilles, et d'hypothèses simplificatrices sur la disposition du feuillage (pas d'azimut et pas de superposition privilégiée des feuilles).

La fonction $\mu$ (i) pour la hauteur de visée $1=35^{\circ}$, prend des valeurs très voisines, de l'ordre de 0,9 quels que soient les types de géométrie du couvert (LEMEUR, 1970).

BonHOMмe $(1974,1976)$ montre que l'on peut utiliser la relation suivante pour calculer l'inđice foliaire, $f$, d'une végétation à partir de $s_{1}=35^{\circ}$ :

$$
f( \pm 10 \%)=-2,58 \log _{10} s_{1}=35^{\circ}
$$

(f est la surface de feuilles par unité de surface de sol).

Dans les cas de couverts végétaux où les hypothèses simplificatrices sont vérifiées, Bonномme (1974) trouve une bonne corrélation entre l'estimation de l'indice foliaire de cultures et sa mesure directe sur le terrain. De même, l'auteur démontre sur des couverts théoriques (de WIT, 1965 ; LEMEUR, 1970) que l'erreur due à l'existence d'azimuts privilégiés des feuilles reste faible, pour les cultures.

Dans le cas où il y a des superpositions privilégiées, il est nécessaire d'étalonner l'indice foliaire mesuré in situ, avec le logarithme de la surface de trouées à une hauteur angulaire égale à 35 degrés.

\subsection{Protocole de prise des clichés hémisphériques sur le terrain}

Pour éviter les taches de lumière les photographies sont prises dans la journée, par ciel totalement nuageux, ou tôt le matin et tard le soir par ciel uniforme tant que les rayons du soleil n'éclairent pas le sommet de la voûte du Chêne pubescent. 
Elles ont été réalisées en huit emplacements, dans chacune des quatre stations, le long de la ligne horizontale de trente-deux mètres, située dans laxe central et longitudinal de la station (respectivement 2, 6, 10, 14, 18, 22, 26 et 30 mètres) et en 5 ou 6 niveaux verticalement (sol; $1,5 \mathrm{~m}$ ou $2 \mathrm{~m} ; 3 \mathrm{~m} ; 4 \mathrm{~m} ; 4,5 \mathrm{~m} ; 6 \mathrm{~m}$ et $7 \mathrm{~m}$ ). L'appareil photographique était monté à l'extrémité d’un mât télescopique. L'horizontalité de l'objectif était vérifiée par deux niveaux à bulle ronds, un disposé sur l'objectif, l'autre sur un support horizontal fixé sur le mât à un mètre de hauteur.

\section{2. - Méthodes de mesure de la quantité de feuillage à l'aide de points-quadrats optiques}

\subsection{Modèle}

Il s'agit de trouver une méthode se rapprochant de la méthode du point-quadrat couramment utilisée dans les milieux herbacés (Levy \& Madden, 1947; Goodall, 1952) et qui soit applicable dans les milieux forestiers où les systèmes mécaniques sont très difficiles à mettre en place.

MAC Arthur \& Horn (1969) ont décrit une méthode de point-quadrat optique permettant de reconstituer des profils de feuillage dans la forêt. Un appareil photographique de type réflex muni d'un verre dépoli réticulé érigeant des lignes verticales virtuelles est utilisé. Dans les forêts à voûte basse, inférieure à quinze mètres, et en plusieurs emplacements dans la forêt, les auteurs effectuent des mesures de distances aux premières feuilles qui interceptent la grille de points. Pour les voûtes plus hautes, ils estiment la proportion de ciel non obscurci sur l'écran de l'objectif.

Dans cette étude, la méthode de Mac Arthur \& Horn a été reprise en utilisant le stratiscope décrit par Blondel \& Cuvilier (1977). L'appareil se compose d'un objectif de $120 \mathrm{~mm}$ de focale, d'un prisme de renvoi d'image à $90^{\prime \prime}$ et d'un oculaire de $64 \mathrm{~mm}$ de focale.

MAC ARThur \& Horn (1969) estiment la quantité de feuillage Q(h) entre deux niveaux verticaux $h_{1}$ et $h_{.2}$, à partir de la distribution des distances aux premières feuilles; ils en déduisent que :

$$
\mathrm{Q}(\mathrm{h})=-\log _{\mathrm{n}}\left\{\gamma\left(\mathrm{h}_{1}\right) / \gamma\left(\mathrm{h}_{2}\right)\right\} \text {, avec } \mathrm{h}_{1}>\mathrm{h}_{2}
$$

$\gamma\left(h_{1}\right)$ est estimé par le nombre de contacts trouvés au-dessus de la hauteur $h_{1}$.

$\gamma\left(\mathrm{h}_{2}\right)$ est estimé par la même façon. $\mathrm{Q}(\mathrm{h})$ exprime le nombre moyen de contacts avec des feuilles le long de lignes de visée verticales imaginaires. La démarche mathématique utilisée par MAC ARTHUR \& HORN, qui ne sera pas reprise dans cet article, suppose que l'on émet deux hypothèses simplificatrices sur la disposition des feuilles dans la voûte :

1) Il y a indépendance entre niveaux de contacts possibles. C'est-à-dire que la probabilité de trouver un contact à une certaine hauteur sur une ligne de visée est indépendante des réalisations qui pourraient se produire au-dessous de ce niveau sur cette ligne de visée (plusieurs contacts avec des feuilles ou contact avec le ciel). 
2) Il n'y a qu'un contact par niveau ; ce qui est très imparfaitement réalisé dans notre cas, étant donné l'imprécision sur l'évaluation des distances.

La voûte foliaire à analyser doit être dépourvue de clairière. En effet, près des bords de la clairière, la répartition des feuilles ne vérifie certainement pas ces deux hypothèses. Pourtant, dans une étude récente sur des forêts caducifoliées américaines, ABER (1979) conclut que cette méthode ne procure pas de bonnes estimations de l'indice foliaire total, mais qu'elle permet d'obtenir dans une station des profils verticaux de distribution relative du feuillage bien corrélés avec la distribution verticale de l'indice foliaire.

\subsection{Protocole de lecture sur le terrain des contacts aux premières feuilles}

L'objectif photographique est étalonné de manière à estimer des distances verticales aux premières feuilles à partir de deux mètres de hauteur et de cinquante centimètres en cinquante centimètres jusqu'à sept mètres puis de mètre en mètre au-delà. L'appareil est muni d'un verre dépoli avec un réticule comportant un point central. La mise au point de l'objectif se fait sur la feuille qui est en contact avec ce point.

L'appareil est positionné sur un tripode à un mètre du sol. Les lectures se font en déplaçant l'appareil le long de la ligne horizontale de trente-deux mètres.

Le nombre de points de lecture dépend de la densité de feuilles dans la voûte et/ou de la hauteur de la voûte. Dans le premier cas, il est nécessaire d'obtenir un nombre suffisant de contacts à découvert; dans le second cas, le nombre de niveaux où des feuilles risquent de se trouver étant plus élevé, il est nécessaire d'échantillonner un grand nombre de points.

Aber (1979), note que la quantité de feuillage est fortement influencée par le nombre de points de lecture mais surtout par le nombre de points à découvert.

A la Rouvière 2, nous avons réalisé 320 points de lecture horizontalement (tous les dix centimètres sur la ligne de trente-deux mètres). Il en résulte un certain profil de densité de feuillage. De cette répartition des distances aux premières feuilles, nous n'avons retenu que les points distants horizontalement de $20 \mathrm{~cm}$ puis de $50 \mathrm{~cm}$. D'où, trois profils de quantité du feuillage correspondant à 320,160 et 64 lectures (cf. figure 1).

En utilisant le test du $\chi^{2}$, ces trois profils ne sont pas différents, au seuil de signification 0,05 .

Pour les voûtes assez basses (inférieures à $10 \mathrm{~m}$ ) nous n'avons effectué que 64 points de lecture; à Murviel, où la voûte atteint environ 13 mètres, nous avons lu 160 points.

Postérieurement, nous avons réalisé 576 points de lecture dans les quatre stations (les données ne sont pas comparables puisqu'elles n'ont pas été recueillies la même année). Les résultats présentés dans le tableau 2 montrent que la quantité de feuillage au-dessus de $2 \mathrm{~m}$ de hauteur dans chacune des stations est évaluée assez correctement avec 64 points et qu'elle ne varie pas de manière significative 
avec l'augmentation du nombre de points de lecture. Cette quantité de feuillage est liée à la proportion de contacts avec des troncs qui ne sont pas pris en compte dans l'analyse et à la part de contacts à découvert. Ces deux quantités sont sensiblement les mêmes de 64 à 576 points de lecture.

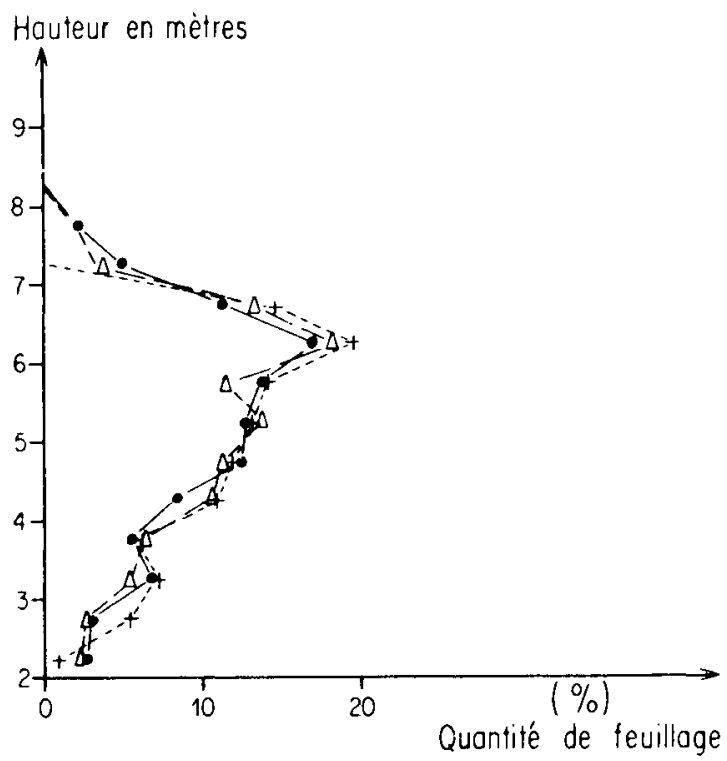

FIG. 1

Comparaisons de profils verticaux de quantité de feuillage dans un taillis de chêne pubescent (La Rouvière 2) des Hautes Garrigues du Montpelliérais réalisés à l'aide de points-quadrats optiques

Comparisons of foliage - height profiles in a Quercus pubescens coppice (La Rouvière 2) of the Montpelliérais region, as measured by the optical point quadrats method

(+) : avec 64 points de lecture de la distance à la première feuille with 64 reading points of the height to the first leaf

$(\triangle)$ : avec 160 points de lecture de la distance à la première feuille with 160 reading points of the height to the first leaf

(. ) : avec 320 points de lecture de la distance à la première feuille with 320 reading points of the height to the first leat

\section{3. - Méthode radiométrique}

\subsection{Principe}

L'absorption sélective des voûtes forestières a été démontrée depuis longtemps (Shirley, 1929 ; Seybold, 1936 ; Egle, 1937 ; Vezina \& Boulter, 1966 ; Federer \& TANnER, 1966). Cette absorption sélective est liée aux variations du facteur 
d'absorption des feuilles avec la longueur d'onde; un minimum relatif d'absorption se produit dans le vert et une grande diminution dans l'infra-rouge, alors que le maximum d'absorption se produit dans le rouge, bleu, violet et ultraviolet (SAUBerer, 1937 ; Moos \& Loomis, 1952 ; Costes, 1960 ; Methy, 1972 ; Wooley, 1971 ; StoutJesdiJK, 1972).

\section{TABleau 2}

Estimations de la quantité totale de feuillage obtenue à l'aide de points-quadrats optiques, en faisant varier le nombre de points de lecture, de la distance à la première feuille, dans quatre taillis de chêne pubescent des hautes garrigues du Montpelliérais

Estimates of total foliage quantity obtained with the optical point quadrats method as a function of total sample size (reading points of the distance to the lowest leaf) in four Quercus pubescens coppices of the Montpelliérais region

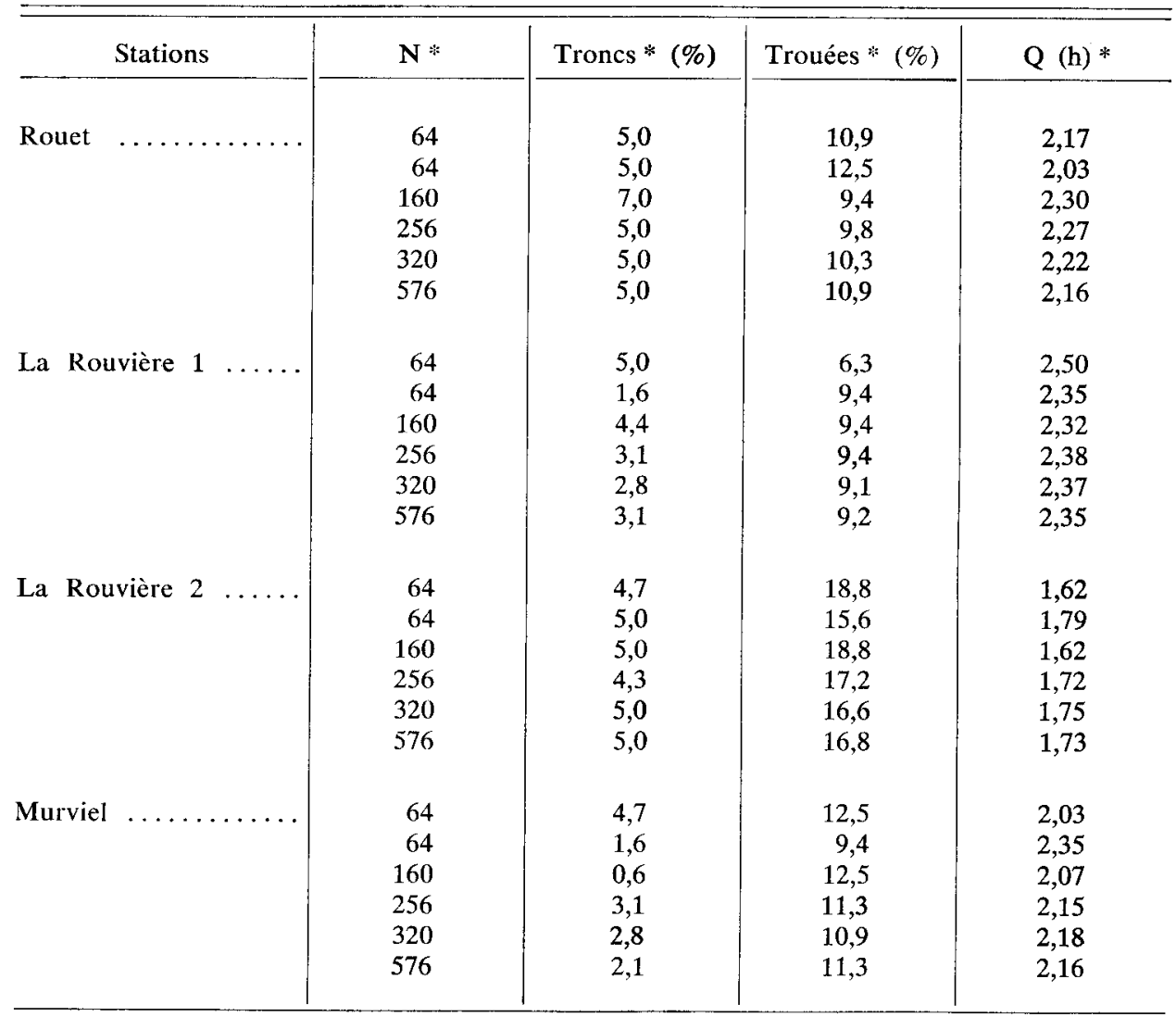

* : - N : Nombre de points de lecture de la distance à la première feuille.

- Troncs (\%) : Proportion de contacts avec des troncs ou des branches.

- Trouées (\%) : Proportion de contacts à découvert.

- $Q$ (h) : Quantité de feuillage qui exprime le nombre moyen de feuilles en contact avec des lignes de visées verticales virtuelles depuis $1 \mathrm{~m}$ jusqu'au sommet de la voâte foliaire. 
De nombreuses méthodes d'évaluation de la production primaire utilisent ces propriétés d'absorption sélective du rayonnement solaire par la végétation. Tucker et al. (1974), MaC Naughton (1976, 1977), Methy (1977) estiment la biomasse de couverts végétaux à partir de la mesure du rapport des facteurs de réflexion relatifs à deux longueurs d'onde, l'une située dans la partie rouge du spectre, l'autre dans la partie infra-rouge. JoRdan (1969) suggère que le rapport des facteurs de transmission pour les longueurs d'onde visible et infra-rouge est un outil utile pour l'estimation de l'indice foliaire dans des forêts tropicales. L'auteur trouve une relation linéaire entre ce rapport et lindice foliaire mesuré directement sur le terrain par Odum et al. (1963). Norman \& Jarvis (1975) ont émis des critiques sur la procédure utilisée par Jordan. Ces auteurs notent qu'elle n'est valable que pour des stations à structure homogène et si le rapport des facteurs de transmission relatifs aux deux longueurs d'onde est calibré in situ avec l'indice foliaire; en particulier, d'une espèce à l'autre la corrélation entre ce rapport et l'indice foliaire ne serait pas la même.

Dans cette étude, nous avons repris lapproche de JoRDaN.

\subsection{Protocole de mesure}

C'est l'appareil mis au point par Methy (1977) qui a été utilisé dans cette étude. Il a subi quelques modifications relatives notamment à son champ de visée rendu égale à 180 degrés.

L'appareil délivre des tensions proportionnelles à chacune des énergies monochromatiques transmises correspondant aux longueurs d'onde 798 et 679 nanomètres. C'est le rapport $\mathrm{K}$ de ces tensions qui a été étudié.

La sélection spectrale des pigments foliaires par temps serein a été vérifiée sous le couvert de chêne pubescent, ainsi que la constance du rapport $K$ à découvert et pendant une journée ensoleillée (Houssard, 1979).

Les conditions de mesure étant très strictes - ciel parfaitement serein, période de mesure très courte dans la journée (période où la hauteur du soleil dépasse 45 degrés) - nous n’avons pu échantillonner que les stations de la Rouvière 1 et la Rouvière 2.

Les mesures ont été effectuées dans ces deux stations en 64 points le long de la ligne horizontale de trente-deux mètres et en neuf niveaux verticaux (respectivement $0 ; 1 ; 2 ; 3 ; 4 ; 4,5 ; 5 ; 6$ et 7 mètres) en chacun des points, l'appareil était monté à l'extrémité d'un mât téléscopique. Elles ont été réalisées au cours du mois de juillet 1977 et pour des hauteurs de soleil supérieures à 45 degrés. Dans ces conditions, on peut supposer qu'il n'y a pas de modifications notables, en fonction de la hauteur du soleil, de la distribution spectrale de l'énergie transmise par la végétation (cf. Метнy, 1974 pour Quercus ilex).

Les valeurs du rapport $K$ ont été corrigées par le cosinus de l'angle entre le soleil et la normale à la surface de référence pour étalonner le rapport $\mathrm{K}$ à l'épaisseur réelle de végétation traversée.

Les valeurs ponctuelles du rapport $\mathbf{K}$ n'ont pas été prises en compte étant donné :

1) l'hétérogénéité du climat radiatif, liée à l'hétérogénéité de la structure de la végétation ; 
2) les variations d'absorption foliaire suivant le milieu, l'âge des feuilles, leur position dans la voûte (Chartier, 1966 ; Kasanaga \& Monsi, 1954).

Seules les valeurs moyennes de $\mathrm{K}$ par niveaux ont été analysées.

\section{4. - Résultats et discussion}

L'indice foliaire estimé à l'aide des photographies hémisphériques a été comparé au rapport $\mathbf{K}$ des tensions proportionnelles aux énergies transmises pour chacune des longueurs d'onde 679 et $798 \mathrm{~nm}$, et à la quantité de feuillage évaluée à l'aide de points-quadrats optiques.

Dans les deux stations où la méthode radiométrique a été testée, la corrélation est très bonne entre le rapport $\mathrm{K}$ et l'indice foliaire estimé, $\mathbf{f}$ :

$\begin{array}{llll}\text { à } & \mathrm{La} \text { Rouvière } 1, \mathrm{f}=6,81 \cdot 10^{-3} & \mathrm{e}^{12,8} \mathrm{~K} & (\mathrm{r}=0,97) \\ \text { à La Rouvière } 2, \mathrm{f}=2,61 \cdot 10^{-4} & \mathrm{e}^{10,6} \mathrm{~K} & (\mathrm{r}=0,99)\end{array}$

Ces deux corrélations sont très proches et la relation liant les valeurs de $\mathbf{K}$ à celles de l'indice foliaire en tenant compte des données des deux stations est (cf. figure 2) :

$$
\mathrm{f}=2.2 \cdot 10^{-3} \mathrm{e}^{10,9 \mathrm{~K}} \quad(\mathrm{r}=0,97)
$$

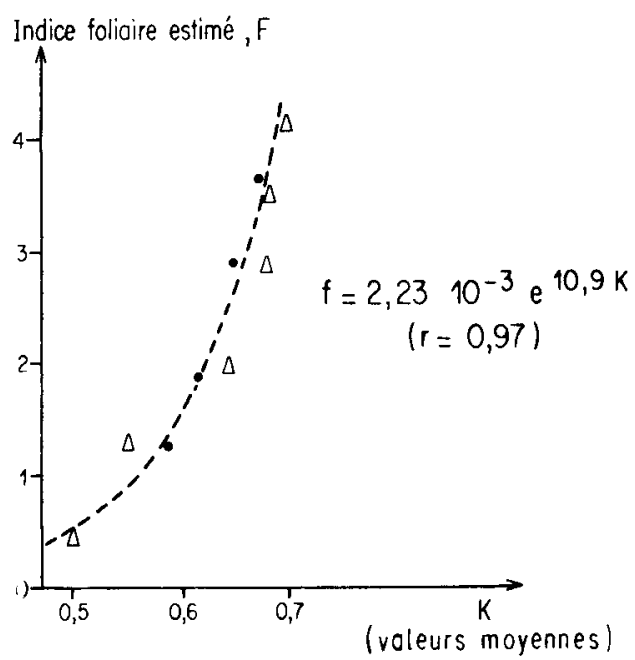

FIG. 2

Comparaison du rapport $K$ mesuré à l'aide d'une méthode radiométrique avec l'indice foliaire estimé par la méthode des photographies hémisphériques

Comparison of $K$ ratio as measured by a radiometric method with estimates of leaf area index achieved with hemispherical photographs

$(\triangle)$ : à La Rouvière $2 ;($ ) : à La Rouvière 1

$K$ est un rapport de tensions proportionnelles à chacune des énergies monochromatiques transmises correspondant aux longueurs d'onde 798 et $679 \mathrm{~nm}$.

$K$ is a ratio of two tensions proportional to spectral intensities of infrared $(798 \mathrm{~nm})$ and red light $(679 \mathrm{~nm})$. 
Le rapport $\mathrm{K}$ est corrélé à la quantité de feuillage, $\mathrm{Q}(\mathrm{h})$, par la relation (cf. figure 3):

$$
\mathrm{Q}(\mathrm{h})=2,15 \cdot 10^{-3} \mathrm{e}^{10,2} \mathbf{K} \quad(\mathrm{r}=0,89)
$$

avec, à La Rouvière 1: $\mathrm{Q}(\mathrm{h})=0,02 \mathrm{e}^{7.2 \mathrm{~K}} \quad(\mathbf{r}=0,99)$

à La Rouvière 2: $\mathrm{Q}(\mathrm{h})=1,85 \cdot 10^{-3} \mathrm{e}^{10,1 \mathrm{~K}} \quad(\mathrm{r}=0,96)$

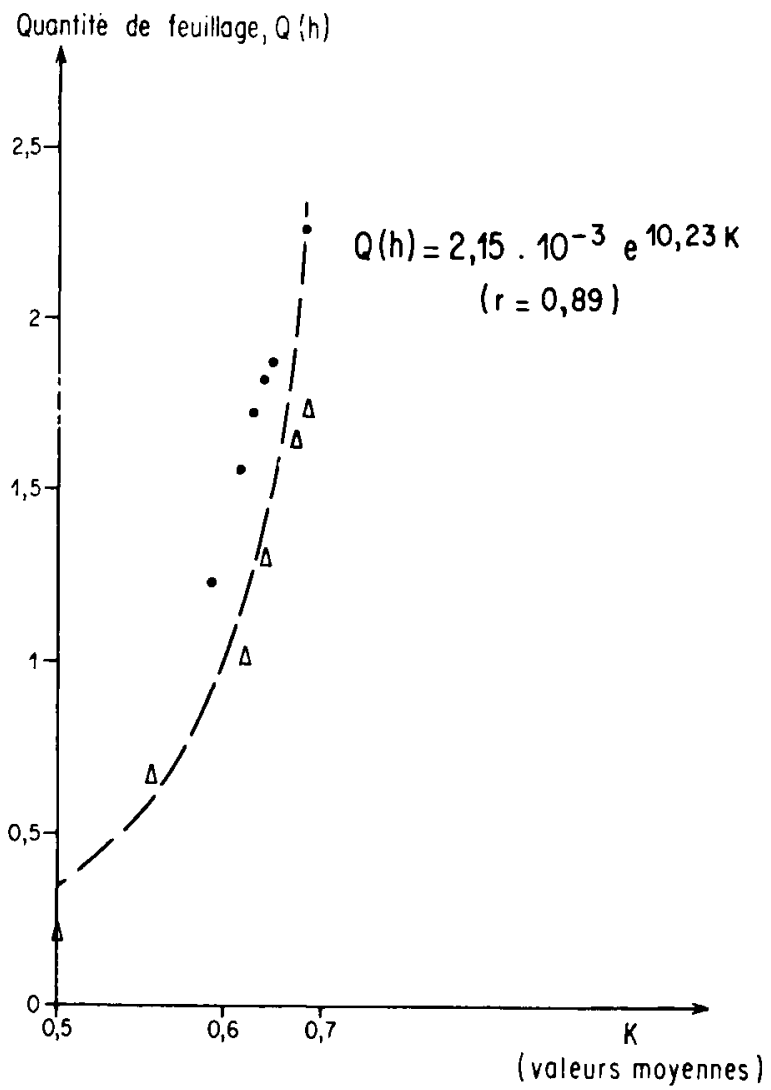

FIG. 3

Comparaison du rapport $K$ mesuré à l'aide d'une méthode radiométrique avec la quantité de feuillage obtenue à l'aide de points-quadrats optiques

Comparison of $K$ ratio as measured by a radiometric method with foliage quantity achieved with the optical point quadrats method

$(\triangle)$ : à La Rouvière 2; ( . ) : à La Rouvière 1

$\mathrm{K}$ est un rapport de tensions proportionnelles à chacune des énergies monochromatiques transmises correspondant aux longueurs d'onde 798 et $679 \mathrm{~nm}$.

$K$ is a ratio of two tensions proportional to spectral intensities of infrared $(798 \mathrm{~nm})$ and red light $(679 \mathrm{~nm})$. 
La bonne corrélation existant, dans une station, entre les valeurs moyennes du rapport $\mathrm{K}$ et l'indice foliaire ou la quantité de feuillage, permet d'accorder une signification biologique à ce rapport. Cependant, dans le cas de la relation entre le rapport $\mathrm{K}$ et la quantité de feuillage (cf. figure 3), il semble y avoir un effet dû à la station : les points correspondants à La Rouvière 1 et à La Rouvière 2 sont distincts. En particulier, à La Rouvière 1, le rapport $K$ est inférieur dans tous les niveaux à celui de La Rouvière 2 ; pourtant la quantité de feuillage est plus élevée à La Rouvière 1 . Ce biais pourrait s'expliquer par la saturation du rapport $\mathrm{K}$ au-delà d'une certaine quantité de feuillage. Cependant, l'effet de station semble moins net lorsqu'on compare le rapport $\mathrm{K}$ avec l'indice foliaire estimé (cf. figure 2).

La généralisation des résultats devrait être testée dans un plus grand nombre de stations de chêne pubescent; si l'effet de station se confirmait, la méthode radiométrique deviendrait inutilisable puisqu'il faudrait étalonner le rapport $\mathbf{K}$ à la quantité de feuillage dans chacune des stations.

Il est aussi possible, et nous allons le voir par la suite, de mettre en cause les estimations de l'indice foliaire ou de la quantité de feuillage.

L'indice foliaire estimé, f, est lié à la quantité de feuillage, $\mathrm{Q}(\mathrm{h})$, par la relation (cf. figure 4 et tableau 3 ) :

$$
\begin{gathered}
\mathrm{f}=1,65 \mathrm{Q}(\mathrm{h})^{0,68} \quad(\mathbf{r}=0,95) \\
\left(\text { et } \mathrm{Q}(\mathrm{h})=0,52 \mathrm{f}^{1,33}\right)
\end{gathered}
$$

En ce qui concerne cette corrélation on est amené à faire deux observations :

1) si l'on considère que l'indice foliaire estimé doit être égal à la quantité de feuillage, puisqu'on est censé mesurer la même quantité, il y a une sous-estimation de la quantité de feuillage par rapport à l'indice foliaire, ou inversement une surestimation de l'indice foliaire par rapport à la quantité de feuillage (exception faite pour Rouet);

2) les points correspondants à chacune des stations se distinguent sur la figure 4 et semblent appartenir à des corrélations différentes.

Le nombre de points de lecture avec la méthode des points-quadrats optiques ne semble pas influencer significativement la valeur de la quantité de feuillage (cf. tableau 2).

Par contre, on peut considérer avec ABER (1979) et à la suite de WARrEN WILson (1958) que l'échantillonnage des points à la verticale entraîne une sous-estimation de la quantité de feuillage puisque les feuilles ne sont pas toutes disposées dans le plan horizontal, ou parce qu'il y a des agrégations privilégiées de feuilles dans ce plan.

D'autre part, à Murviel l'indice foliaire est très largement supérieur à la quantité de feuillage. On peut supposer qu'il y a eu surestimation de l'indice foliaire due au protocole de prise des clichés hémisphériques. En effet, cette station présente une voûte très haute $(\simeq 12 \mathrm{~m})$. La base du houppier de Chêne pubescent est comprise entre 5 et $6 \mathrm{~m}$ de hauteur (cf. tableau 1). Le dernier niveau de prise des clichés $(7 \mathrm{~m})$ se situe à la limite de la base du houppier et les clichés pris dans les niveaux inférieurs doivent présenter de petites trouées étant donné la distance de l'objectif au houppier. Il en résulte une surestimation de l'indice foliaire (les trouées sont petites, 
leur estimation est moins précise). C'est l'inverse à Rouet qui présente une voûte très basse $(<8 \mathrm{~m})$. A l'aide du mât télescopique, la formation est explorée sur toute sa hauteur, les trouées sont plus grandes et l'indice foliaire pourrait être sousestimé.

\section{TABLEAU 3}

Quantité de feuillage et indice foliaire estimé à plusieurs niveaux dans quatre taillis de chêne pubescent

Foliage quantity and estimates of leaf area index at various levels in four Quercus pubescens coppices

\begin{tabular}{|c|c|c|}
\hline Stations et niveaux de mesure & $\begin{array}{c}\text { Quantité } \\
\text { de feuillage }\end{array}$ & $\begin{array}{c}\text { Indice foliaire } \\
\text { estimé }\end{array}$ \\
\hline \multicolumn{3}{|l|}{ Rouet } \\
\hline 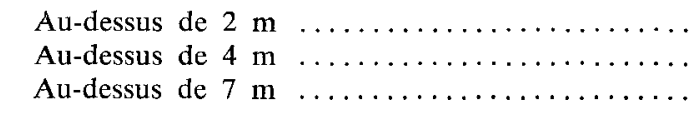 & $\begin{array}{l}2,55 \\
2,05 \\
0,02\end{array}$ & $\begin{array}{l}2,75 \\
2,15 \\
0,001\end{array}$ \\
\hline \multicolumn{3}{|l|}{ La Rouvière 1} \\
\hline 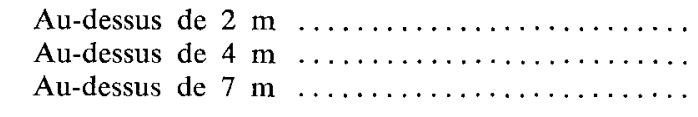 & $\begin{array}{l}2,55 \\
1,89 \\
1,22\end{array}$ & $\begin{array}{l}3,65 \\
2,89 \\
1,26\end{array}$ \\
\hline \multicolumn{3}{|l|}{ La Rouvière 2} \\
\hline $\begin{array}{l}\text { Au-dessus de } 1,5 \mathrm{~m} \\
\text { Au-dessus de } 4,5 \mathrm{~m} \\
\text { Au-dessus de } 7 \\
\text { m }\end{array}$ & $\begin{array}{l}1,75 \\
1,11 \\
0,28\end{array}$ & $\begin{array}{l}3,47 \\
1,96 \\
0,43\end{array}$ \\
\hline \multicolumn{3}{|l|}{ Murviel } \\
\hline 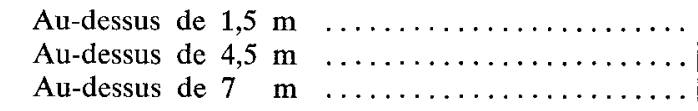 & $\begin{array}{l}2,06 \\
1,80 \\
1,49\end{array}$ & $\begin{array}{l}5,13 \\
4,05 \\
3,13\end{array}$ \\
\hline
\end{tabular}

La Rouvière 1 et 2 ont sensiblement la même hauteur de voûte ( 8 et $9 \mathrm{~m}$ ). La Rouvière 1 présente un sous-bois très encombré par des arbustes, alors que La Rouvière 2 a un sous-bois herbacé. La densité de tiges est supérieure à La Rouvière 1 (cf. tableau 1). On devrait logiquement s'attendre à ce qu'à La Rouvière 1, l'indice foliaire estimé soit très largement supérieur à la quantité de feuillage (fort encombrement, petites trouées). Ce devrait être l'inverse à La Rouvière 2. Or pour une même quantité de feuillage, on obtient un indice foliaire supérieur à La Rouvière 2 et inversement, pour un même indice foliaire, la quantité de feuillage est supérieure à La Rouvière 1 (cf. figure 4). 


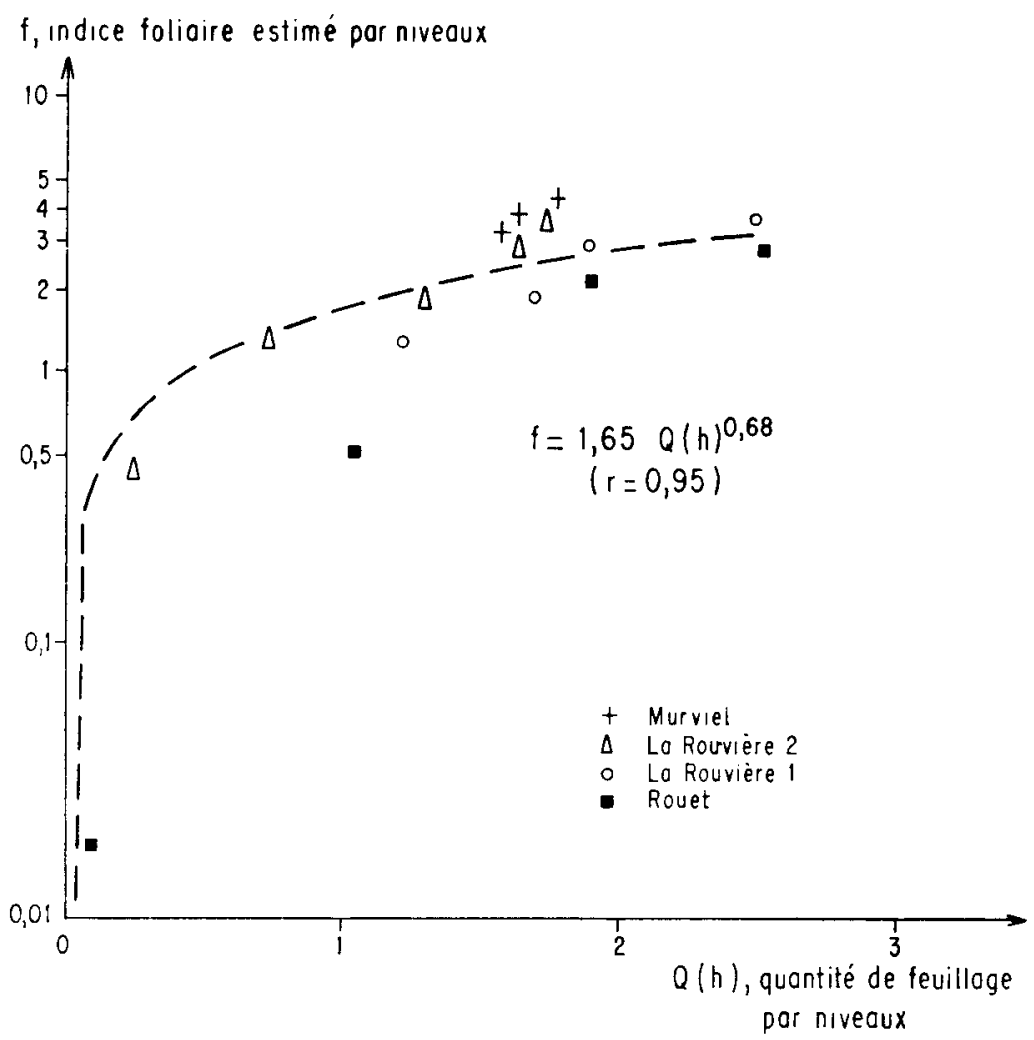

Fig. 4

Comparaison de l'indice foliaire estimé à l'aide de photographies hémisphériques, avec la quantité de feuillage évaluée à l'aide de points quadrats optiques, dans quatre taillis de chêne pubescent et en plusieurs niveaux verticaux dans chaque station

Comparison of estimates of leaf area index achieved with hemispherical photographs, with foliage quantity as measured with the optical point quadrats method, in four Quercus pubescens coppices and at various levels in each stand

Des erreurs dans les temps d'exposition au moment de la prise des clichés dans une station pourraient être mises en cause. Ces erreurs sont généralement imputables aux différences de luminosité d'un point à l'autre du ciel. Elles conduisent à de mauvaises estimations du pourcentage de trouées sur les photographies et donc à de mauvaises estimations de l'indice foliaire.

Si maintenant on considère non plus les valeurs absolues de l'indice foliaire ou de la quantité de feuillage mais leurs valeurs relatives, les deux profils obtenus dans chacune des stations ne sont pas différents avec un test du $\chi^{2}$ au seuil de signification 0,01 (l'indice foliaire est pris comme référence) (cf. figure 5).

En résumé, les valeurs absolues du rapport $K$, de la quantité de feuillage ou de l'indice foliaire sont affectées par la structure de la végétation dans les stations, ou 
Houleur en mètres

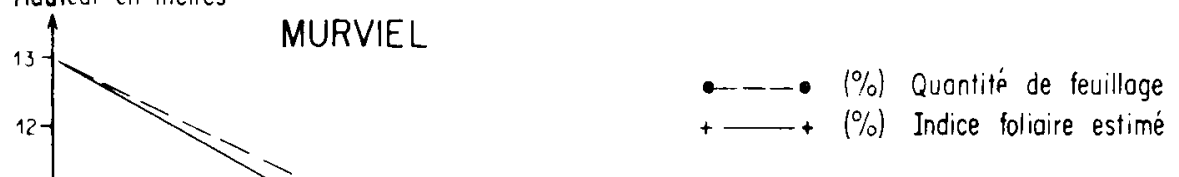

\section{ROUET}
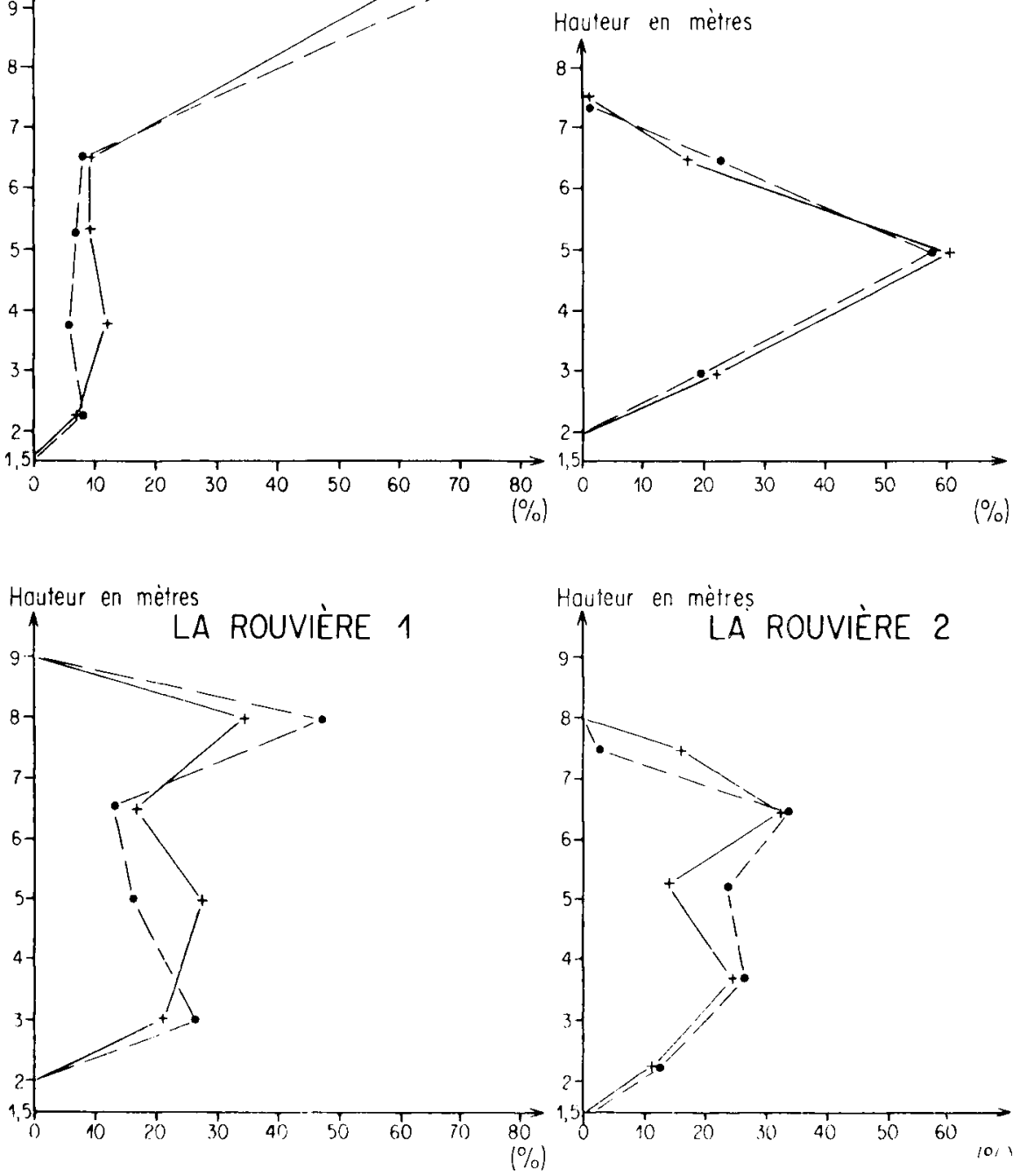

FIG. 5

Comparaison de profils verticaux de quantité de feuillage, (.), évaluée par la méthode des points-quadrats optiques et d'indice foliaire estimé, $(+)$, par les photographies hémisphériques dans quatre taillis de chêne pubescent

Comparison of foliage-height profiles, (.), as measured by the optical point-quadrats method, with estimates of leaf area index profiles, $(+)$, achieved with hemispherical photographs, in four Quercus pubescens coppices 
par les protocoles de recueil des données; mais dans une station les trois méthodes sont corrélées et les profils de lindice foliaire ou de la quantité de feuillage en valeurs relatives sont parfaitement comparables. Le choix d'une méthode fera donc intervenir le coût de recueil des données.

Les photographies hémisphériques ne peuvent être prises qu'en absence de soleil et de vent, soit le soir très tard ou le matin très tôt alors que le soleil n'éclaire pas le sommet de la voûte, ou par ciel nuageux homogène. Ensuite, les films doivent subir un contretypage pour être très contrastés, en ne laissant apparaître que le blanc et le noir. Enfin, la mesure du pourcentage de trouées par couronnes peut se faire à l'aide d'un bras mobile muni de phototransistors (appareil décrit par Bonhomme \& Chartier, 1972 ; et par Ducrey, 1975 a). Cette façon de procéder est la plus rapide mais elle nécessite un appareillage relativement sophistiqué. Une deuxième méthode - tirage en grand format des films, découpage par couronnes, mesure du pourcentage de trouées à l'aide d'un planimètre - demande beaucoup de manipulations.

Chacune des phases de récolte des données à l'aide de cette méthode est donc relativement coûteuse en temps; cependant la photographie constitue un document qu'il est possible d'analyser le moment voulu.

Dans des formations végétales complexes telles que les forêts, et pour des mesures de facteurs de transmission du rayonnement, l'utilisation de la méthode radiométrique n'est pas très aisée; elle nécessite des conditions de ciel parfaitement serein, l'absence de vent et la lecture des deux tensions dans les zones de pénombre est très difficile. Par contre, dans des cultures monospécifiques et denses, et pour des mesures de facteurs de réflexion du rayonnement, cette méthode s'est révélée très utile pour estimer la biomasse au cours de lannée (Methy, 1977).

La méthode des photographies hémisphériques et celle du radiomètre pour construire des profils foliaires en forêt nécessitent l'utilisation d'un mât télescopique qui permet de déplacer en hauteur les instruments de mesure. Cette utilisation est rendue très fastidieuse dans des taillis où la densité de tiges est élevée, et où donc les houppiers sont étroitements imbriqués. Bien souvent, les conditions de mesure (horizontalité et immobilité des appareils) ne sont pas respectées très rigoureusement.

Par contre, avec la méthode des points-quadrats optiques, l'appareil reste toujours à la même hauteur $(1 \mathrm{~m})$, les déplacements se font beaucoup plus facilement. Il nous a fallu environ 4 heures pour réaliser 320 lectures de la distance à la première feuille en effectuant une visée tous les $10 \mathrm{~cm}$. Le protocole peut être amélioré en lisant plusieurs points à chaque déplacement. Ainsi, avec 9 points de lecture par visée, on peut lire facilement 576 points en 2 heures, en déplaçant l'appareil tous les $50 \mathrm{~cm}$.

Cette méthode n'exige que l'absence de vent; elle permet de distinguer les espèces et donc d'obtenir la répartition du feuillage par espèce (ce qui n'est pas possible avec les deux autres méthodes) et ce par niveaux relativement fins $(50 \mathrm{~cm})$. Pour l'établissement de profils verticaux de nappe foliaire en forêt, la méthode des points-quadrats optiques offre donc beaucoup de renseignements et à un moindre coût de recueil des données. 


\section{Remerciements}

Ce travail a été effectué grâce au contrat D.G.R.S.T. No N567, N633, N674 : «Structure, dynamique et utilisation des formations à chêne pubescent en zone bioclimatique méditerranéenne », et à un programme du C.N.R.S. intitulé «Chêne pubescent ».

R. Bonhomme (I.N.R.A., Versailles) et M. DuCrey (C.N.R.F., Champenoux) nous ont aidé à comprendre et à mettre en place sur le terrain la méthode des photographies hémisphériques. M. Ducrey nous a permis au C.N.R.F. de lire les photographies et de traiter les résultats à l'ordinateur. M. Methy (C.E.P.E., Montpellier) nous a aidé et conseillé en ce qui concerne la méthode radiométrique qu'il a mise au point. M. Terrouane (Faculté des Lettres, Montpellier) a analysé le modèle mathématique sous-jacent à la méthode de Mac Arthur et Horn. Enfin, J. Blondel (Institut de Botanique, Montpellier) nous a prêté le stratiscope et donné conseil pour son emploi.

Nous sommes très reconnaissants envers ces personnes pour l'aide qu'elles nous ont apportée.

\section{Summary}

Measurement of relative foliage quantity by strata in Quercus pubescens Willd. coppices

Three methods for estimating foliage quantity by height in forest are described, and results obtained with it compared.

These are (1) a method using hemispherical photographs for estimating leaf-area index, (2) an optical point-quadrat method for estimating foliage quantity by height, (3) a radiometric method which uses selective absorption of radiation by leaf pigments : two tensions proportional to spectral intensities of infra-red $(798 \mathrm{~nm})$ and red $(679 \mathrm{~nm})$ light are measured and expressed as a ratio, $\mathrm{K}$; this ratio is calibrated with leaf area index or foliage quantity.

They have been tested in four Quercus pubescens coppices in the «Hautes Garrigues du Montpelliérais » (South of France).

Estimates of leaf area index, foliage quantity or $\mathrm{K}$ value are affected by vegetation structure or by sampling methods. But in a stand, results obtained with the three methods are correlated, and profiles of leaf area index or of foliage quantity are similar.

The optical point-quadrat method furnishes more informations than the others and is the faster method for estimating foliage quantity by height in forest.

\section{Références bibliographiques}

ABER J.D., 1979. A method for estimating foliage-height profiles in broad-leaved forests. J. Ecol., 67, 35-40.

ANDERson M.C., 1966. Stand structure and light penetration. II. - A theoretical analysis. J. appl. Ecol., 3, 41-54.

Blondel J. et Cuvillier R., 1977. Une méthode simple et rapide pour décrire les habitats d'oiseaux : le stratiscope. Oikos, 29 (2), 326-331.

Blondel J., Ferry C, et Frochot B., 1973. Avifaune et végétation, essai d'analyse de la diversité. Alauda, 41, 63-84. 
Bonнomme R., 1974. Détermination de profils d'indice foliaire et de rayonnement dans un couvert végétal à l'aide de photographies hémisphériques faites in situ. Thèse de Doc. Ing., Univ. Aix-Marseille, $30 \mathrm{p}$.

Bonhomme R., 1976. Détermination des profils d'indice foliaire et de rayonnement dans un couvert végétal à l'aide de photographies hémisphériques faites in situ. Ann. agron., 27 (1), 33-59.

Bonhomme R., et Chartier P., 1972. The interpretation and automatic measurement of hemispherical photographs to obtain sunlit foliage and gap frequency. Isr. J. agric. Res., 22 (2), 53-61.

Braun-Blanquet J., 1936. La chênaie méditerranéenne. Comm. S.I.G.M.A., 45.

Braun-Blanquet J., Roussine N., Negre R. et Emberger L., 1952. Les groupements végétaux de la France méditerranéenne. C.N.R.S. et Direction de la Carte des Groupements Végétaux de l'Afrique du Nord, $297 \mathrm{p}$.

CaChan P., 1963. Signification écologique des variations microclimatiques verticales dans la forêt de basse Côte-d'Ivoire. Ann. Fac. Sci. Univ. Dakar, 8, 89-155.

Chartier P., 1966. Etude du microclimat lumineux dans la végétation. Ann. agron., 17, 571-602.

CoDY M.L., 1968. On the methods of ressource division in grassland bird communities. Am. Nat., 102, 107-147.

Costes C., 1960. Absorption et utilisation de l'énergie lumineuse par les végétaux cultivés dans des conditions naturelles. Ann. Physiol. vég., 2, 176-229.

Ducrey M., 1975a. Utilisation des photographies hémisphériques pour le calcul des perméabilités des couverts forestiers au rayonnement solaire. I. - Analyse théorique de l'interception. Ann. Sci. for., 32 (2), 73-92.

Ducrey M., 1975b. Utilisation de photographies hémisphériques pour le calcul des perméabilités des couverts forestiers au rayonnement solaire. II. - Etude expérimentale. Ann. Sci. for., 32 (4), 205-221.

EgLe K., 1937. Zur kenntnis des lichtfeldes der pflanze und der blättfarbstoffe. Planta, 26, 546-583.

Federer C.A. et Tanner C.B., 1966. Vertical distribution of light in the forest. Ecology, 47, 555-560.

FERNANDEZ R., 1978. Les peuplements de chêne pubescent des Hautes Garrigues du Montpelliérais, étude dendrométrique et écologique. Mémoire E.N.I.T.E.F., 41 pages + annexes.

Ford E.D. et Newbould P.J., 1971. The leaf canopy of a coppiced deciduous woodland. J. Ecol., 59, 843-862.

Goodall D., 1952. Some consideration on the use of point-quadrats for the analysis of vegetation. Aust. J. sci. Res., Série B, 5, 1-41.

Harcombe P.A. et Marks P.L., 1977. Understory structure of a mesic forest in Southeast Texas. Ecology, 58, 1144-1151.

Houssard C., 1979. Etude de la structure de taillis de Chêne pubescent (Quercus pubescens Willd.). Exemples pris dans les Hautes Garrigues du Montpelliérais. Thèse $3^{c}$ cycle, U.S.T.L. Montpellier.

JoRDAN C.J., 1969. Derivation of leaf area index from quality of light on the forest floor. Ecology, 50, 663-666.

Kasanaga H. et Monsi M., 1954. On the light transmission of leaves and its meaning for the production of matter in plant communities. Jpn J. Bot., 14, 304-324.

Kira T., Shinozaki K. et Hozumi K., 1969. Structure of forest canopies as related to their primary productivity. Plant Cell Physiol., 10, 129-142. 
LEDENT J.F., 1974. Une formule générale pour le calcul de la surface foliaire soumise au rayonnement solaire direct. Biométr.-Praxim., 14, 69-75.

LEDENT J.F., 1976. Comment interpréter les fréquences de contact dans l'analyse des surfaces photosynthétiques par la méthode des points-quadrats, spécialement dans le cas d'une distribution binômiale négative. Biométr.-Praxim., 16, 37-49.

LEmeur R., 1970. Effects of spatial leaf distribution on penetration and interception of direct radiation. Symp. on Plant Response to Climate Factors, U.N.E.S.C.O., Uppsala, 349-356.

Levy E.B. et Madden E.A., 1933. The point method of pasture analysis. N.Z.J. Agric., 46, 267-279.

Mac ARThur R.H. et Horn H.S., 1969. Foliage profile by vertical measurements. Ecology, 50, $802-804$.

Mac Arthur R.H. et Mac Arthur J.W., 1961. On bird species diversity. Ecology, 42, 594-598.

Mac Arthur R.H., Recher H. et Cody M., 1966. On the relation between habitat selection and species diversity. Am. Nat., 100, 319-332.

Mac Naughton S.J., 1976. Serengeti migratory wildebeest: Facilitation of energy flow by grazing. Science, 191, 92-94.

MaC Naughton S.J., 1977. Diversity and stability of ecological communities : a comment on the role of empiricism in ecology. Am. Nat., 111, 515-525.

Methy M., 1972. Optical properties of leaves : Methods and measurements techniques. Isr. J. agric. Res., 22, 77-84.

MeThy M., 1974. Interception du rayonnement solaire par différents types de végétation dans la région méditerranéenne. Thèse U.S.T.L., Montpellier, 56 p. + annexes.

Methy M., 1977. Estimation quantitative de la biomasse d'un peuplement de graminées par une méthode optique non destructive. Oecol. Plant., 12 (4), 395-401.

Miller P.C., 1967. Leaf orientation and energy exchange in quaking aspen and Gambell'oak in central Colorado. Oecol. Plant., 2, 241-270.

Miller P.C., 1969. Test of solar radiation models in three forest canopies. Ecology, 50, 878-885.

Moen A.N., 1973. Wildlife ecology. W.H. Freeman and co. (éd.), San Francisco, 458 p.

Monsi M. et Saeki T., 1953. Über den Lichtfaktor in den Pflanzengesellschaften und seine Bedeutung für die stoffproduction. JPn. J. Bot., 14, 22-52.

Moos R.A. et Loomis W.E., 1952. Absorption spectra of leaves. I. - The visible spectrum. Plant Physiol., 27, 370-391.

Nilson T., 1971. A theoretical analysis of the frequency of gaps in plant stands. Agric. Meteorol., 8, 25-38.

Nouman J.M. et Jarvis P.G., 1975. Photosynthesis of Sitka Spruce (Picea Sitkensis [Bong.] Carr.). V. - Radiation penetration theory and a test case. J. appl. Ecol., 12 (3), 839-877.

Odum H.T., Copeland B.J. et Brown R.Z., 1963. Direct and optical assay of leaf mass of the lower montane rain forest of Puerto Rico. Proc. natl. Acad. Sci. U.S.A., 49, 429-434.

Sauberer F., 1937. Zur Kenntnis der Strhlungsverhaltnisse in Pflanbestanden. Bioklim. Beibl., 4, $145-155$.

Seybold A., 1936. Ueber des Licht Faktor Photophysiologische Prozesse. Jber. wiss. Bot., 82, 741-795.

SHIRLEY H.L., 1929. The influence of light intensity upon the growth of plants. Am. J. Bot., 16, 354-390. 
StoutJesdijk Ph., 1972. Spectral transmission curves of some types of leaf canopies with a note on seed germination. Acta bot. neerl., 21 (3), 185-191.

Tucker C.J., Miller L.D. et Pearson R.L., 1974. Measurements of the combined effect of green biomass, chlorophyll and leaf water in canopy spectroreflectance of the short grass prairie, pp. 601-627, in Proceedings of the Second Annual Remote Sensing of Earth Research Conference, F. Sharoki (éd.), University of Tennessee Press, Tullahoma.

Vezina P.E. et Boulter D.W.K., 1966. The spectral composition of near ultraviolet and visible radiation beneath forest canopies. Can. J. Bot., 44, 1267-1284.

Warren Wilson J., 1958. Analysis of the spatial distribution of foliage by two-dimensional points-quadrats. New Phytol., 59, 92-101.

WARREN Wilson J., 1959. Influence of spatial arrangement of foliage area in grassland pp. 51-61, in The measurement of grassland productivity, J.D. Ivins (éd.), London.

WARREN Wilson J., 1960. Inclined point-quadrats. New Phytol., 59, 1-18.

WARREN Wilson J., 1963. Estimation of foliage denseness and foliage angle by inclined point-quadrats. Aust. J. Bot., 11, 95-105.

WARREN Wilson J., 1967. Stand structure and light penetration. III. - Sunlit foliage area. J. appl. Ecol., 4, 159-166.

WeBs W.N., 1948. Environmental analysis of a winter deer range. Trans. N. Am. Wildl. Conf., 13, 442-450.

Wit C.T. de, 1965. Photosynthesis of leaf canopies. Agric. Res. Rep. Wageningen, 63, 1-57.

Woolley J.T., 1971. Reflectance and transmittance of light by leaves. Plant Physiol., 47, 656-662. 